\title{
Nuevos desafíos para cuestiones pedagógicas históricas. La evaluación en contexto de pandemia
}

\author{
New challenges for historical pedagogical questions. Assessment \\ in the context of a pandemic
}

Daniela Sala

https://orcid.org/0000-0002-0777-0281

saladaniela@gmail.com

Facultad de Trabajo Social I Universidad Nacional

de La Plata | Argentina

\section{RESUMEN}

Ante el pasaje abrupto de clases presenciales a virtuales en la Universidad Nacional de La Plata, dentro del sorpresivo contexto de pandemia, surgen interrogantes y aspectos que parecen nuevos pero que en realidad están basados en dilemas históricos. Uno de ellos es la evaluación.

Les estudiantes se preguntan ¿cómo me van a evaluar? ¿qué actividades son obligatorias? ¿cómo me van a poner una nota? ¿cómo hago para no perder el año?, etc. Y les docentes también nos cuestionamos cómo evaluar, ¿nuestras propuestas de enseñanza virtual están facilitando los aprendizajes?, ¿hay que poner una calificación numérica a cada trabajo?, ¿cómo comprobar que les estudiantes estén aprehendiendo los contenidos mínimos de la asignatura para acreditarla?

\section{ABSTRACT}

Faced with the abrupt transition from face-to-face to virtual classes at the National University of La Plata, within the surprising context of a pandemic, questions and aspects appear that seem new but are actually based on historical dilemmas. One of them is evaluation.

The students wonder how they are going to evaluate me? What activities are compulsory? How can I get a grade? How can I avoid missing the year? Etc. And teachers also ask ourselves how to evaluate, are our virtual teaching proposals facilitating learning? Is it necessary to put a numerical grade on each job? How to verify that students are learning the minimum content of the subject to accredit it?

PALABRAS CLAVE

desafíos, evaluación, virtualización, pandemia
KEY WORDS

challenges, evaluation, virtualization, pandemic 


\section{PRESENTACIÓN}

Ante el pasaje abrupto de clases presenciales a virtuales en la Universidad Nacional de La Plata, dentro del sorpresivo contexto de pandemia, surgen interrogantes y aspectos que parecen nuevos pero que en realidad están basados en dilemas históricos. Uno de ellos es la evaluación.

Para comprender el debate sobre evaluación en la virtualidad, debemos primero aclarar que no es lo mismo un curso pensado para ser a distancia que una materia virtualizada en este contexto, con el objetivo de garantizar el derecho a la educación de les estudiantes.

En general comenzamos contactando y conociendo a nuestres estudiantes, intentando llevar adelante el programa sin muchos cambios, en parte por la urgencia, también por desconocimiento. Les mismes estudiantes nos fueron planteando desde comentarios y memes la necesidad de otro tipo de abordaje y tratamiento de los contenidos; la combinación de estrategias y plataformas; la añoranza por los encuentros cara a cara y los debates dentro del aula.

La preocupación por la desigualdad en el acceso, la conectividad y el equipamiento, que parecía saldada con la posibilidad de uso de computadoras e internet en las unidades académicas, resurgió con mucha fuerza al tener que estudiar/trabajar cada persona desde su casa. Incluso recuperando la necesidad de aprender y enseñar, al mismo tiempo, la utilización de las TIC con fines académicos. Porque que la mayoría de nuestres estudiantes sean jóvenes no nos garantiza que tengan experiencia en el envío de trabajos por mail, o participar en un foro de un aula virtual, por nombrar ejemplos de usos básicos. Y estos aspectos, hoy más que nunca se pueden convertir en expulsores, si les 
estudiantes se sienten abandonades a su suerte. Por lo tanto es necesario considerarlos parte de los contenidos a enseñar.

Posteriormente surgió la pregunta por la evaluación. Les estudiantes se preguntan ¿Cómo me van a evaluar? ¿Qué actividades son obligatorias? ¿Cómo me van a poner una nota? ¿Cómo hago para no perder el año?, etc. Y les docentes también nos cuestionamos cómo evaluar: ¿Nuestras propuestas de enseñanza virtual están facilitando los aprendizajes? ¿Hay que poner una calificación numérica a cada trabajo? ¿Cómo comprobar que les estudiantes estén aprehendiendo los contenidos mínimos de la asignatura para acreditarla?

Les estudiantes se preguntan ¿Cómo me van a evaluar? ¿Qué actividades son obligatorias? ¿Cómo me van a poner una nota? ¿Cómo hago para no perder el año?, etc. Y les docentes también nos cuestionamos cómo evaluar: ¿Nuestras propuestas de enseñanza virtual están facilitando los aprendizajes? ¿Hay que poner una calificación numérica a cada trabajo? ¿Cómo comprobar que les estudiantes estén aprehendiendo los contenidos mínimos de la asignatura para acreditarla?

\section{PRIMEROS PASOS EN LA VIRTUALIZACIÓN DE CLASES}

En un primer momento la principal preocupación de les docentes estuvo enfocada a incluir a todes les estudiantes en el aula virtual y construir un vínculo pedagógico, ya que la suspensión de clases coincidió con el inicio de las mismas. Resultó sumamente importante el contacto con todes les estudiantes inscriptes para su matriculación al aula virtual como así también conocer los motivos por los cuales no podían hacerlo o utilizarla en su plenitud.

Aquí, algunes estudiantes nos han planteado que tienen problemas de conectividad (sin WiFi en el domicilio) y falta de equipamiento (sin computadora, solo con el celular; o con computadora de uso compartido con otres estudiantes convivientes); pero también dificultades por no saber usar las TIC, especialmente para el aprendizaje, el cansancio de leer en la pantalla, no estar dentro del aula web de la materia por haber pedido la matriculación en otra, etc. Estas cuestiones se van abordando en forma personalizada por las cátedras y la Dirección de Inclusión Educativa de la Facultad de Trabajo Social mediante un seguimiento a través de correo electrónico, redes sociales e incluso WhatsApp. También plantearon otros inconvenientes como quienes siguen concurriendo a sus lugares de trabajo y vivencian situaciones estresantes o quienes trabajan desde sus hogares, con mayor cansancio por estar haciendo muchas actividades a la vez, pues se suma la reorganización familiar y por ejemplo, la necesidad de acompañar las actividades educativas de quienes tienen hijes. 
algunes estudiantes nos han planteado que tienen problemas de conectividad (sin WiFi en el domicilio) y falta de equipamiento (sin computadora, solo con el celular; o con computadora de uso compartido con otres estudiantes convivientes); pero también dificultades por no saber usar las TIC, especialmente para el aprendizaje, el cansancio de leer en la pantalla, no estar dentro del aula web de la materia por haber pedido la matriculación en otra, etc.

Les docentes del Profesorado en Trabajo Social (reunión virtual del 28/04/20) refieren que la gran mayoría de les estudiantes están participando de las propuestas virtuales, aunque se observa mayor entrega de trabajos prácticos que de participación en foros, esto puede deberse a la forma en que se plantean las consignas, las ideas acerca de la obligatoriedad de las diferentes actividades, la similitud o diferencia con actividades conocidas, etc.

La circulación de numerosos memes (nueva forma de comunicación que se basa en lo gráfico y el humor para analizar el momento que estamos viviendo) sobre el bombardeo de "pdf" que sintieron les estudiantes al comienzo de la cursada, especialmente aquellos que plantean que no están aprendiendo, nos lleva a reflexionar sobre la forma en que se está planteando la enseñanza. Por un lado, el mantra que repetimos acerca de la imposibilidad de trasladar de la misma forma una clase presencial a lo virtual, pero también nos lleva a revisar los fundamentos pedagógicos de nuestras propuestas en general. Retomando a Freire (1970) sobre la importancia de abandonar la idea de educación bancaria, como depósito de información, que hoy pueden obtener les estudiantes en internet. Pasar a la construcción situada, crítica y colaborativa de conocimientos.

En este sentido, resulta enriquecedor escuchar a personas que vienen investigando sobre la educación mediada por la tecnología como Mariana Maggio (2020) de la UBA, quien nos plantea "\#NoAIRevoleo de PDF" y propone pensar en un currículum minimalista, donde identifiquemos pocos núcleos fuertes, que les estudiantes tengan que aprender y que sean posibles de enseñar en este contexto, que recuperen las tendencias culturales (series) y se logre la creación de conocimiento original. También plantea que la clase debe ser una experiencia que valga la pena ser vivida e incluso en la virtualidad las propuestas inspiren y emocionen.

Al comenzar el Seminario Educación en Entornos Virtuales del Profesorado en Trabajo Social, realizamos un relevamiento con nuestres estudiantes, a modo de evaluación diagnóstica sobre sus conocimientos e ideas previas sobre dichos entornos de aprendizaje. Un aspecto que nos llamó la atención fue la valoración positiva del aula web (90\%), especialmente como facilitadora de la continuidad del estudio y la posibilidad de organizarse el tiempo, es decir la flexibilidad que tiene la educación virtual a los tiempos de cada estudiante. El aspecto 
que más extrañan les estudiantes es el encuentro entre compañeres, los debates e intercambios en las clases, cuestión que el $10 \%$ de les encuestades no logran realizar en las aulas virtuales.

Un aspecto que nos Ilamó la atención fue la valoración positiva del aula web (90\%), especialmente como facilitadora de la continuidad del estudio y la posibilidad de organizarse el tiempo, es decir la flexibilidad que tiene la educación virtual a los tiempos de cada estudiante.

Esto nos interpela a les docentes acerca del tipo de recursos y consignas que estamos proponiendo. La sugerencia de especialistas es variar las actividades, combinando producciones individuales y grupales, pero sobre todo estimulando la reflexión personal y creatividad, donde puedan generarse debates, cuestionamientos, comentarios entre compañeres, que superen ampliamente la producción y devolución individual de un trabajo práctico (cuestión también necesaria). Pensar propuestas desafiantes, que no se puedan resolver copiando y pegando de un texto o de la web. Carina Lion (2020) habla de gamificación en cuanto a recuperar el placer por aprender mediante juegos que nos involucren y nos incentiven a investigar, generar hipótesis, ensayar respuestas e incluso fortalezcan la comunicación cuando requieran la elaboración de estrategias en forma colectiva y en lugar de castigar el error, promuevan la exploración e integración de contenidos. Hay experiencias de elaboraciones muy creativas de juegos al estilo de "Preguntados" o "Scaperoom" con los contenidos de las materias, que vale la pena conocer.

A esto se suman los encuentros sincrónicos que se están llevando a cabo en algunas materias a través de diferentes plataformas (especialmente Zoom, Jitsi y Webex), los cuales han aportado al conocimiento mutuo, intercambio y la disminución de ansiedades e incertidumbre de les estudiantes. Si bien es imposible que el $100 \%$ de les estudiantes se pueda conectar en forma sincrónica, dichos encuentros se graban y luego suben al aula web para ser vistos por todes. Pudiendo ser utilizados tanto para la explicación de temas, como para la devolución de producciones de estudiantes, exposiciones grupales, espacios de consulta y sobre todo intercambios tanto acerca de ideas, dudas y reflexiones sobre los contenidos, como también el fortalecimiento del vínculo pedagógico y lazo social entre estudiantes, ya que el saber cómo están transitando la cuarentena contiene y permite situar las propuestas de enseñanza a la realidad de les estudiantes.

Otra cuestión muy valorada por les estudiantes es la producción de videos explicativos por parte de las cátedras (video de clase, presentación audiovisual comentada, etc.) Si bien es algo que están comenzando a realizar varias cátedras, les docentes plantean el mayor tiempo que implica su elaboración. 

ción de videos explicativos por parte de las cátedras (video de clase, presentación audiovisual comentada, etc.)

Este es un aspecto a tener en cuenta, la sensación de mayor exigencia, pareciera que estamos trabajando las 24 hs, no sólo en la elaboración de materiales para la clase (escritos o audiovisuales) y consignas que conllevan más horas de trabajo, también la corrección de trabajos, devoluciones generales e individualizadas, participación en foros, respuesta a consultas que ingresan a cualquier hora, lo que se suma a la capacitación en el uso de los entornos virtuales, las exploraciones mediante ensayo y error, las consultas a colegas con mayor experiencia, las tensiones que generan el uso de herramientas desconocidas, la autoexigencia de mostrar propuestas acabadas sin el tiempo previo necesario para la planificación.

Fue diferente la situación de las materias que ya venían utilizando un aula virtual como aula extendida, complementaria a las clases presenciales. El conocimiento previo de la plataforma AulasWeb de UNLP y la dinámica propia de la enseñanza virtual, las encontró en mejores condiciones para afrontar el trabajo plenamente en la virtualidad.

\section{¿Por qué la evaluación es un tema que incomoda? Algunos aportes para revertirlo}

Pareciera que la evaluación es una instancia disociada del aprendizaje, como aquel momento en que les estudiantes deben demostrar haber adquirido los aprendizajes propuestos por la materia, en forma individual, apelando más a la memoria que al pensamiento crítico. Donde los nervios pueden jugar una mala pasada y hacerles perder el año/cuatrimestre. Incluso como acto autoritario donde la mirada docente es la única válida.

Con esto en mente, desarrollé en 2014 como Trabajo Final Integrador de la Especialización en Docencia Universitaria un sistema de evaluación integral (bajo la modalidad de proyecto de innovación pedagógica) que permitiera a les estudiantes reflexionar críticamente sobre su proceso de aprendizaje, incluyendo la participación de los diferentes actores involucrades: estudiantes (en forma individual y como miembre de un grupo), sus docentes y les referentes institucionales de las prácticas. Procurando generar un sistema de evaluación democrático, participativo, integral, formativo, motivador y orientador, basado en la evaluación cualitativa.

que según diversos autores (Álvarez Méndez, 2000; Araujo, 2009) está basada en el paradigma fenomenológico-constructivista y tiene las siguientes características, rasgos y principios: es formativa, continua, procesual, holística, integradora; describe e interpreta para comprender; es democrática y participativa; explicita y negocia los 
criterios; pretende ser justa, honesta, válida, confiable, de calidad, comprometida, responsable y equitativa; está integrada a las tareas de aprendizaje; con triangulación de fuentes y metodológica; y es flexible, abierta a lo no previsto. (Sala, 2016)

En este sentido, resulta fundamental diferenciar evaluación de acreditación. La evaluación es un proceso mucho más amplio que poner una nota que implique la aprobación o no de una materia. Abarcando desde la recuperación de los saberes previos de les estudiantes (evaluación diagnóstica), la mirada crítica a los procesos de enseñanza y aprendizaje, para poder mejorarlos (evaluación formativa), hasta la comprobación del logro de los objetivos de la materia y su aprobación (evaluación sumativa).

Una buena evaluación es una herramienta de conocimiento, está enmarcada en la enseñanza de aprendizajes significativos y los desafíos cognitivos solicitados deben ir trabajándose durante las clases y complejizándose progresivamente para favorecer las mejores producciones de les estudiantes. De esta manera los errores deben ser tomados como fuente de información, comprender su naturaleza, origen o causa posibilita a les docentes mejorar sus propuestas de enseñanza de tal manera que les estudiantes potencien sus procesos cognitivos (Álvarez Méndez, 2000; Celman, 1998; Litwin, 2008; Bain, 2007). (Sala, 2016)

Por lo tanto, el examen no es la única forma de evaluación y el aula virtual permite tener el registro de todo el proceso de cada estudiante mediante diferentes intervenciones y producciones. En este aspecto también la combinación de estrategias será lo que permita una mejor evaluación, por ejemplo estimular la autoevaluación y evaluación de pares, actividades evaluadas grupales e individuales, orales y escritas, etc. Esto conlleva mayor implicación de les estudiantes en su proceso de aprendizaje y que la evaluación sea más integral.

Como refiere Marilina Lipsman (2020) la evaluación puede ser invisible y pública al mismo tiempo, invisible porque se va realizando en las mismas actividades de aprendizaje y pública como instancia que externaliza los conocimientos para la acreditación y al mismo tiempo permite validarla.

Por otra parte, la explicitación de las formas y criterios de evaluación es algo que tranquiliza a les estudiantes, conocer las reglas del juego, y aporta a la autoevaluación.

En cuanto a la calificación numérica, para que deje de ser un misterio, se les puede facilitar a les estudiantes una escala con anterioridad a la entrega de un trabajo integrador/evaluación parcial o final, como la siguiente: 


\begin{tabular}{|c|c|}
\hline Calificación & Características de la respuesta \\
\hline Desaprobado & $\begin{array}{l}\text { Respuesta desacertada: conceptos muy confusos } \\
\text { que no apuntan al tema de la consigna o con erro- } \\
\text { res importantes. Quien lo lee no logra reconstruir, a } \\
\text { partir de lo escrito, una respuesta plausible para lo } \\
\text { que se pregunta. El texto o bien trata sobre otro tema } \\
\text { con relación a la consigna, o bien intenta centrarse } \\
\text { en lo que se pide, pero no lo logra por escasez de co- } \\
\text { nocimiento sobre ese tema. Los problemas de orga- } \\
\text { nización pasan a un segundo plano, ya que son los } \\
\text { contenidos los que no se relacionan con la consigna } \\
\text { o están tratados de forma incorrecta. }\end{array}$ \\
\hline 4 & $\begin{array}{l}\text { Respuesta no enfocada hacia lo que se pregunta: se } \\
\text { responde utilizando más o menos una serie de con- } \\
\text { ceptos, pero que aportan en escasa medida a explicar } \\
\text { lo que solicita la consigna. Presenta serios problemas } \\
\text { en su organización: no se provee un inicio o cierre y } \\
\text { predominan las ideas desconectadas entre sí, ocul- } \\
\text { tándose las relaciones conceptuales que mantienen } \\
\text { entre ellas. Hay conceptos confusos. La persona que } \\
\text { lo lee debe hacer un gran esfuerzo para comprender } \\
\text { lo que está escrito. }\end{array}$ \\
\hline 5,6 & $\begin{array}{l}\text { Respuesta sin jerarquizar: aborda lo que se pre- } \\
\text { gunta, pero sin ponerlo en primer plano ya que pre- } \\
\text { senta los conceptos pertinentes en el mismo nivel } \\
\text { que otros no directamente pertinentes. Tiene pro- } \\
\text { blemas en la organización ya que o bien no se pro- } \\
\text { vee un inicio o cierre, o bien aparecen ideas desco- } \\
\text { nectadas o poco claras. Se entiende lo que se explica, } \\
\text { pero con cierto esfuerzo por parte quien lee. }\end{array}$ \\
\hline 7,8 & $\begin{array}{l}\text { Respuesta bien enfocada: aborda explícitamente } \\
\text { lo que pide la consigna, es decir, jerarquiza las ideas } \\
\text { que explican lo que se solicita. Desarrolla en forma } \\
\text { correcta los conceptos pertinentes. Presenta una } \\
\text { buena organización, la respuesta se centra en expli- } \\
\text { car lo que se pide desde el comienzo, y en su desarro- } \\
\text { llo amplía esta explicación. Facilita la tarea de quien } \\
\text { lee porque las ideas están expresadas claramente y } \\
\text { ligadas entre sí. }\end{array}$ \\
\hline 9,10 & $\begin{array}{l}\text { Respuesta a las consignas con elaboración propia: } \\
\text { que demuestra comprensión de los textos, con acti- } \\
\text { tud analítica, crítica, creativa. Da gusto leerlo. }\end{array}$ \\
\hline
\end{tabular}

Basada en la Escala de evaluación de exámenes escritos de Paula Carlino $(2005,135-136)$, utilizada por la Cátedra Trabajo Social III- FTS 
Otra estrategia de evaluación es la construcción de rúbricas, donde se enumeran criterios de evaluación, con sus diferentes escalas, por ej

\begin{tabular}{|l|l|l|l|l|}
\hline Categoría & \multicolumn{1}{|c|}{$\mathbf{4}$} & \multicolumn{1}{|c|}{$\mathbf{3}$} & $\mathbf{2}$ & \multicolumn{1}{|c|}{$\mathbf{1}$} \\
\hline Redacción & $\begin{array}{l}\text { No hay errores } \\
\text { de gramática, } \\
\text { ortografía o } \\
\text { puntuación. }\end{array}$ & $\begin{array}{l}\text { Casi no hay } \\
\text { errores de } \\
\text { gramática, } \\
\text { ortografía o } \\
\text { puntuación. }\end{array}$ & $\begin{array}{l}\text { Unos pocos } \\
\text { errores de } \\
\text { gramática, } \\
\text { ortografía } \\
\text { o puntuación. }\end{array}$ & $\begin{array}{l}\text { Muchos errores } \\
\text { de gramática, } \\
\text { ortografía } \\
\text { o puntuación. }\end{array}$ \\
\hline Análisis & $\begin{array}{l}\text { Realiza un aná- } \\
\text { lisis crítico de } \\
\text { la información } \\
\text { obtenida }\end{array}$ & $\begin{array}{l}\text { Reflexiona so- } \\
\text { bre la informa- } \\
\text { ción obtenida }\end{array}$ & $\begin{array}{l}\text { Describe con } \\
\text { sus palabras } \\
\text { los datos } \\
\text { obtenidos }\end{array}$ & $\begin{array}{l}\text { Corta y pega } \\
\text { información }\end{array}$ \\
\hline
\end{tabular}

MyRubic

Y por último quisiera mencionar los portfolios, que son una carpeta (virtual) donde les estudiantes recuperan algunos trabajos realizados durante la cursada que den cuenta de su aprendizaje. Brinda la posibilidad a les estudiantes de elegir cuáles quieren mostrar, porque los considera sus mejores producciones o evidencian progresos y los acompañan con una reflexión metacognitiva, es decir que justifique por qué los eligió y pueda autoevaluarse (los logros y cuestiones pendientes, estrategias para mejorar, etc.)

\section{Estas herramientas para la evaluación apuntan a la autono- mía de les estudiantes, pero con una guía por parte de les do- centes, que les sirva de andamiaje.}

Estas herramientas para la evaluación apuntan a la autonomía de les estudiantes, pero con una guía por parte de les docentes, que les sirva de andamiaje.

\section{REFLEXIONES FINALES}

La intención de este escrito fue reflexionar sobre la evaluación en el contexto de virtualización de clases por pandemia, afrontando los nuevos desafíos y recuperando la experiencia de la praxis docente en esta temática.

Las tecnologías del aprendizaje y la comunicación nos brindan nuevas herramientas para la evaluación, pero dependerá de nuestro posicionamiento teórico-ideológico su fundamentación y finalidad. Los reportes de las aulas virtuales, por ejemplo, pueden brindarnos información sobre los archivos vistos por les estudiantes, como estrategia de control (sabemos que abrieron un archivo pero nada nos dice sobre la lectura y comprensión del mismo) o de seguimiento para investigar la razones por las que alguien no entrega los trabajos solicitados o los entrega de determinada manera.

Como dice Marilina Lipsman (2013) se puede utilizar la tecnología 
para transparentar procesos cognitivos de estudiantes y revisar el diseño de las clases. Porque así como está más visible el proceso de les estudiantes (todas sus producciones están en el aula) también permite una mejor evaluación del trabajo docente, el análisis del tipo de consignas y materiales propuestos en relación a la respuesta de les estudiantes, las diferentes formas de comunicación estimuladas, la construcción colectiva de conocimiento lograda, etc.

En fin, poner en palabras los miedos e incertidumbres, compartirlos con otres, conocer diferentes experiencias nos fortalece como docentes, fundamenta nuestras decisiones y en definitiva problematiza y mejora nuestras prácticas de enseñanza. 


\section{BIBLIOGRAFIA}

Álvarez Méndez, J. M. (2000) “Evaluación cualitativa: delimitación conceptual y caracterización global" y "Métodos y técnicas de evaluación desde la perspectiva cualitativa", en Didáctica, currículo y evaluación. Ensayos sobre cuestiones didácticas. Buenos Aires: Miño y Dávila

Anijovich, R (comp) (2010). "La evaluación significativa". Buenos Aires: Editorial Paidós

Bain, K. (2007). ¿Cómo evalúan a sus estudiantes y así mismos?", en Lo que hacen los mejores profesores universitarios. Barcelona.

Bertoni, A. Poggi, M. Teobaldo,M. (1995). “La evaluación: nuevos significados para una práctica compleja". Buenos Aires: Ed. Kapelusz.

Carlino, P. (2005). "Escribir, leer y aprender en la universidad. Una introducción a la alfabetización académica". Buenos Aires: Fondo de Cultura Económica de Argentina S.A.

Celman, S. (1998). “ ¿Es posible mejorar la evaluación y transformarla en herramienta de conocimiento?", en Camilloni, A y otras. La evaluación de los aprendizajes en el debate didáctico contemporáneo. Buenos Aires, Paidós Educador.

Freire, Paulo. (1970). “Pedagogía del oprimido”. Montevideo: Tierra Nueva.

Lion, C (2018). "Videojuegos: un prisma para comprender los escenarios culturales contemporáneos". Buenos Aires: Noveduc.

Lion, C (2020) $5^{\circ}$ Webinar "Educación y creatividad en tiempos de coronovirus" 31 de marzo de 2020.Universidad Nacional de Quilmes Virtual y Wikimedia Argentina. Disponible en: https://www.youtube.com/watch?v=zvXzlVghLo4\&list=PLFKNtUouDuswcf7RKmEJpt509ByFWMg81\&index=6\&t=37s

Lipsman, M (2013). “El enriquecimiento de los procesos de evaluación mediados por las TIC en el contexto universitario". En Revista Iberoamericana de Evaluación Educativa, 7(2), 215-222.

Lipsman, M. (2020) $13^{\circ}$ Webinar ¿De qué hablamos cuando hablamos de evaluación en tiempos de distancia social? 5 de mayo de 2020. Universidad Nacional de Quilmes Virtual y Wikimedia Argentina. Disponible en: https://www.youtube. com/watch?v=R6CM9G3ueXw\&list=PLFKNtUouDuswcf7RKmEJpt5O9ByFWMg81\&index $=14 \& \mathrm{t}=0 \mathrm{~s}$

Litwin, E. (2008). “El oficio del docente y la evaluación”, en El oficio de Enseñar. Condiciones y contextos. Buenos Aires: Paidós.

Maggio, M (2020). $2^{\circ}$ Webinar"Educación y creatividad en tiempos de coronovirus" 26 de marzo de 2020. Universidad Nacional de Quilmes Virtual y Wikimedia 
Argentina. Disponible en: https://www.youtube.com/watch?v=Cu1t247HMqQ

Molina, I entrevista a Maggio, M (2020). “Clases virtuales: el desafío es que las plataformas no nos deshumanicen". Publicada el 23 de marzo de 2020 en Revista Red/acción. Recuperado de https://www.redaccion.com.ar/clases-virtuales-el-desafio-es-que-las-plataformas-no-nos-deshumanicen/

Sala, D (2016). “Evaluemos sin miedo. Propuesta de un sistema de evaluación de los aprendizajes de los estudiantes, en las prácticas de formación profesional en la Facultad de Trabajo Social". En Revista Trayectorias Universitarias. Vol 02. $\mathrm{N}^{\circ}$ 02. UNLP 